\title{
Modified Atkins Diet and Low Glycemic Index Treatment for Medication- Resistant Epilepsy: Current Trends in Ketogenic Diet
}

Tomohiro Kumada*, Tomoko Miyajima, Ikuko Hiejima, Fumihito Nozaki, Anri Hayashi and Tatsuya Fujii

Department of Pediatrics, Shiga Medical Center for Children, Shiga, Japan

\begin{abstract}
Compared to the classical ketogenic diet (KD), the modified Atkins diet (MAD) and the low glycemic index treatment (LGIT) are more liberal and less restrictive diet therapies for the treatment of medication-resistant epilepsy. The MAD was first reported by Kossoff et al. in 2003, and gained global popularity with sufficient evidence in its efficacy reported recently in a controlled study. The LGIT was first reported by Pfeifer et al. in 2005 , and its use has also become widespread. We reviewed the efficacy of both diet therapies in the treatment of medication-resistant epilepsy based on the evidence from previous literatures and our own clinical experience. From our experience, the LGIT was more liberal, tolerable, and dietetically balanced than the MAD. To find out which of these diets is most appropriate in different epilepsies and different patients, future controlled comparative studies on the efficacy, tolerability, and dietetic advantages between the MAD and the LGIT are necessary.
\end{abstract}

Keywords: Ketogenic diet; Modified Atkins diet; Low glycemic index treatment; Epilepsy

\section{Introduction}

The ketogenic diets (KDs) have been used for the treatment of medication-resistant epilepsy since the 1920s. The classical KD is a highly restrictive diet with a 3:1-4:1 ketogenic ratio, calculated as the weight of fat divided by that of carbohydrates plus protein. Although the classical KD result in at least a 50\% reduction in seizure frequency in approximately half of epileptic patients, some patients cannot tolerate the diet over a long period of time because of its restrictiveness. Consequently, the modified Atkins diet (MAD) and the low glycemic index treatment (LGIT) that are more liberal and less restrictive than the classical KD, were developed in the 1990-2000s [1,2]. Contrary to the classical $\mathrm{KD}$, these diets can be provided without restriction of calories, protein and fluid intake, and calculation of ketogenic ratio is not necessary. Figure 1 shows the distribution of major nutrients in calories in each diet $[1,2]$. MAD and LGIT have a similar composition pattern and the percentage of fat is less than in KD. These new KDs weigh greater importance in having a stable blood glucose level than the production of ketone bodies.

Because the KDs including MAD and LGIT are the therapy that the patients and parents must prepare by themselves, the menus of the KDs are greatly affected by their food culture. Thus, the practical menus of these KDs differ among countries based on various local food cultures although the concepts of these KDs are common globally. For example, Japanese patients and parents cannot cook daily KDs menus by referring to the textbooks on the KDs in Western countries because the available foods and the tastes are different between Japan and Western countries. Therefore, we have designed the MAD and LGIT menus which Japanese patients and parents can easily prepare and resemble to normal Japanese ethnic menus.

In this paper, we describe the history of development of the KDs, their methods and the evidence of clinical efficacy from the previous literatures. In particular, we present how Japanese patients and parents can cook the MAD and LGIT menus using Japanese ethnic foods concretely in the section of 'dietary concept, compositon, and menu' of each diet. We also describe our own clinical experience in each of these diets, and discuss which of these KDs (MAD or LGIT) should be selected in different patients.

\section{History of the KDs from fasting to the LGIT}

An overview of the history of the KDs shows that the focus has changed from a diet that produces ketone bodies efficiently, which mimics fasting, to one that is palatable and can therefore be followed with high compliance. From the period of Hippocrates, it has been known that seizures can be treated by fasting [3]. The KD protocols consisting of high fat and low carbohydrate were designed to mimic the metabolic effect of fasting. In 1921, Geyelin reported to the American Medical Association that the beneficial effect of fasting continued even after the fast was broken. Then, Wilder theorized that the benefit of the diet depends on the ketonemia observed by Geyelin [3,4]. He showed that ketosis occurred when the ratio of fatty acids to glucose was $>2: 1$. From this theory, the classical $\mathrm{KD}$ was developed and it was widely used in the treatment of epilepsy. Huttenlocher developed a medium chain triglyceride diet in an attempt to make the classical KD more palatable. With the advancement of the pharmacotherapy for epilepsy, however, the KD treatment has gradually become replaced by antiepileptic drugs, particularly after the introduction of carbamazepine and valproic acid in the 1970-80s.

A revival boom of the KDs occurred in the 1990s, starting with an NBC Dateline report in 1994. Soon after, the television movie, "First, Do No Harm", starring Meryl Streep, which was a dramatization of the life of a patient with epilepsy, turned the public's eye to the diet in 1997. In the last decade, the KDs became popular globally; the $1^{\text {st }}$ international symposium on dietary treatments for epilepsy and other neurological disorders was held in 2008 , and the international consensus recommendation was published in Epilepsia in 2009 [5].

*Corresponding author: Dr. Kumada, Department of Pediatrics, Shiga Medical Center for Children, Moriyama, Japan, Tel: +81 77582 6200; Fax: +81 77582 6304; E-mail: tkumada@mccs.med.shiga-pref.jp

Received March 13, 2013; Accepted June 13, 2013; Published June 20, 2013

Citation: Kumada T, Miyajima T, Hiejima I, Nozaki F, Hayashi A, et al. (2013) Modified Atkins Diet and Low Glycemic Index Treatment for Medication-Resistant Epilepsy: Current Trends in Ketogenic Diet. J Neurol Neurophysiol S2: 007 doi:10.4172/2155-9562.S2-007

Copyright: ( 2013 Kumada T, et al. This is an open-access article distributed under the terms of the Creative Commons Attribution License, which permits unrestricted use, distribution, and reproduction in any medium, provided the original author and source are credited. 


\section{Regular Diet}

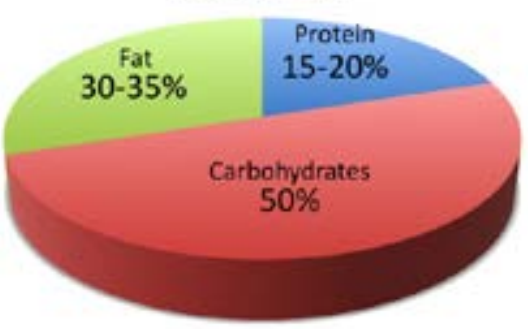

MAD

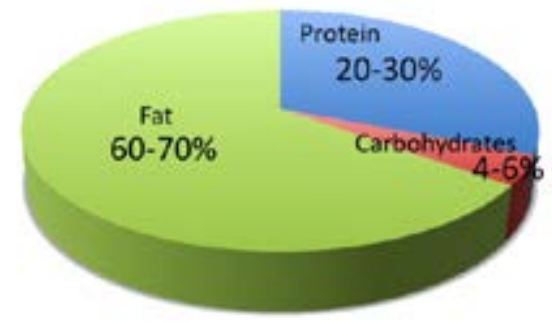

Classical KD



LGIT

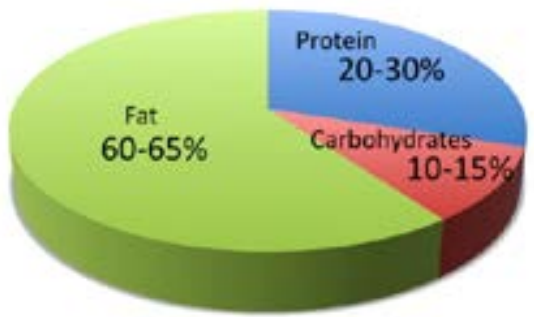

Figure 1: Percentage of carbohydrate, fat, and protein in the regular diet, the classical KD, the MAD, and the LGIT.

Meanwhile, Kossoff from Johns Hopkins University Hospital designed and reported on a new and more liberal KD called 'MAD' in 2003 [6]. Since then, numerous articles on MAD have been published from all over the world, including USA [7,8], Denmark [9,10], France [11], Belgium [12], Korea [13,14], India [15], Iran [16], and Japan [17]. Separately, in 2005, Pfeifer from Massachusetts General Hospital developed and reported on an alternative ketogenic diet, 'LGIT', which was more palatable than the MAD [18]. The diet has also gained a widespread attention. Currently, the MAD and the LGIT are the main KDs used in therapy especially in adolescent and adult patients [19].

\section{The MAD}

\section{Dietary concept, composition and menu}

The MAD was developed at Johns Hopkins Hospital to create a KD that was more liberal and less restrictive than the classical KD for children who were reluctant to follow the classical KD due to its restrictiveness [1]. Dr. Atkins originally developed the diet in the 1970s as a method for weight reduction for people suffering from obesity. This diet was modified so that the fat intake was increased while the carbohydrate restriction was maintained the same as in the "introduction phase" of the original method. The carbohydrate content of the diet is restricted to $10 \mathrm{~g}$ per day at the initiation of the MAD, and is subsequently maintained at 10-30 g per day. Calories, protein and fluids are not restricted. As shown in (Figure 1), the proportion of calories from fat is decreased in the MAD compared to the classical KD. The amount of calories remained from the decrease in fat are assigned to protein so that the ketogenic ratio resulted in 1:1 to 2:1 in the MAD diet. Figure $2 \mathrm{a}$ and $2 \mathrm{~b}$ shows an example of a lunch menu of the MAD in our hospital. The content of carbohydrates, protein, and fat in this menu was $2.5 \mathrm{~g}, 26.8 \mathrm{~g}$, and $42.8 \mathrm{~g}$ respectively, and the ketogenic ratio was $1.46: 1$, which was lower than that of the classical KD, 3:1 to 4:1.

\section{Evidence of efficacy based on previous literatures}

More than 50 articles on the use of MAD for medication-resistant epilepsy have been published since Kossoff et al. first reported on the diet in 2003, and there is accumulating evidence for its efficacy [5]. Auvin reviewed 7 prospective and 2 retrospective reports, and summarized the efficacy of MAD as follows [20]; the responder rate (the proportion of patients with $>50 \%$ seizure reduction) was $51 / 87$ (59\%) after 1 month, 73/152 (48\%) after 3 months, and 46/119 (39\%) after 6 months of MAD therapy. Chen et al. investigated the long-term efficacy of the MAD and reported that the responder rate was $64 / 87$ (74\%) after 1 month, 36/54 (67\%) after 6 months, 28/35 (80\%) after 12 months, and 8/16 (50\%) after 24 months in intent-to-treat analysis [21]. Recently, a randomized controlled trial of the use of MAD for the treatment of medication-resistant childhood epilepsy published by Sharma et al. [22], showed that the proportion of patients with $>90 \%$ seizure reduction $(30 \%$ vs. $7.7 \%, \mathrm{p}=0.005)$ and that with $>50 \%$ seizure reduction ( $52 \%$ vs. $11.5 \%, \mathrm{p}<0.001$ ) was significantly higher in the MAD group than in the control group after 3 months. A number of published reports have shown the efficacy of the MAD in specific epilepsy syndromes and underlying diseases including infantile spasms [15], absence epilepsy [23], juvenile myoclonic epilepsy [24], SturgeWeber syndrome [25], and nonconvulsive status epilepticus [26].

\section{Our experience}

In our group, we have been treating medication-resistant epilepsy with the MAD since 2007. Upon admission, the MAD was initiated with a restriction of the carbohydrate content of the diet to $10 \mathrm{~g}$ per day, and the total calorie content was adjusted to match the patients' usual caloric intake before the diet. After 3 to 4 weeks on the diet, we let the parents decide whether or not to continue the diet. We then follow the patients after discharge every 1-3 months at the out-patient clinic.

In the current report, we summarized data from 16 patients who started the MAD in our hospital. Table 1 shows the profile and shortterm efficacy and tolerability after 3 months on the diet in all of the patients, including 10 patients reported elsewhere [17]. Eight patients could not continue the diet for more than 3 months. Four of them, 

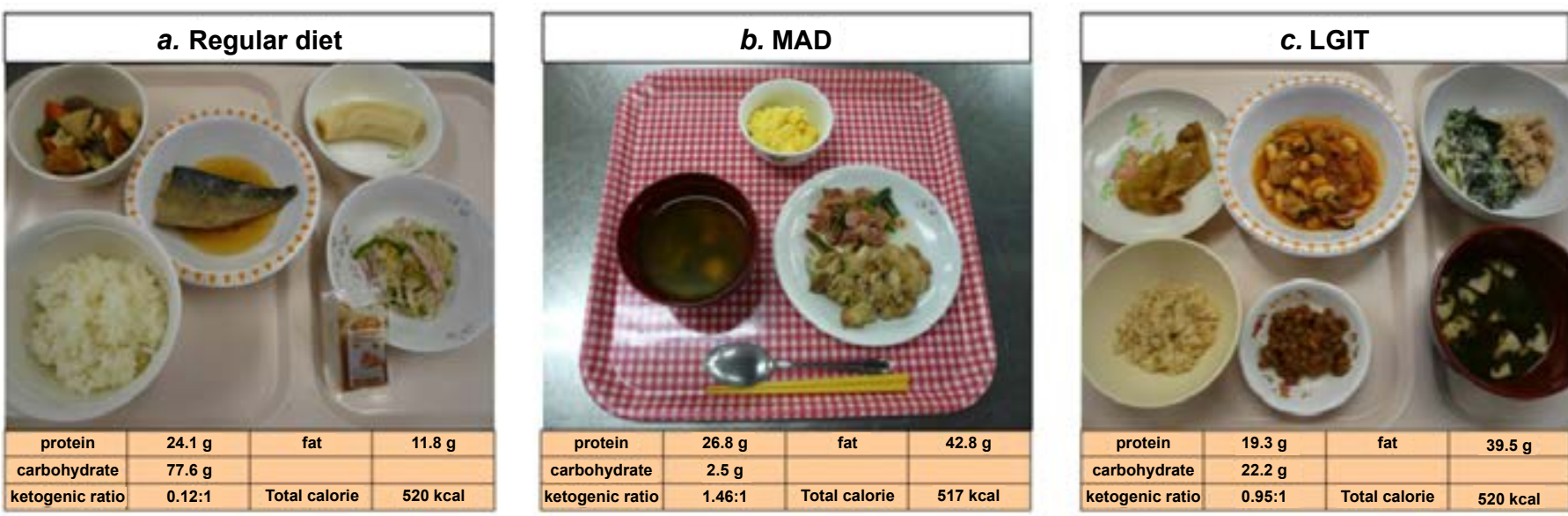

Figure 2: Examples of a lunch menu for the regular diet, the MAD, and the LGIT Each style of lunch menu was provided to case-13 at our hospital. A regular diet which she ate before the start of the MAD at 11 years of age. The lunch consisted of polished rice, boiled mackerel, salad with bean sprouts, nimono (boiled dish), and banana. The ketogenic ratio was $0.12: 1$. This is an example of a diet usually preferred by Japanese. $b$. A MAD menu which she ate during MAD therapy at 11 years of age. The lunch consisted of sauteed chicken with green beans and bacon, scrambled eggs with mayonnaise, and miso (soybean paste) soup. The ketogenic ratio was 1.46:1. c. A LGIT menu which she ate during the LGIT at 13 years of age. The lunch consisted of unpolished rice with natto, pork beans, tuna salad, miso soup, and sauteed chicken. The ketogenic ratio was 0.95:1.

including one who was infected with viral gastroenteritis, refused the menus. In the remaining 4 patients, their families gave up preparing the MAD after discharge. Excluding these drop-outs, the efficacy at 3 months was as follows: 3 individuals were excellent responders (seizure-free), 1 was a good responder ( $>50 \%$ seizure reduction), and the diet was not efficacious in the remaining 4 . The values of serum beta-hydroxybutyrate were elevated over $2000 \mathrm{umol} / \mathrm{L}$ at 3 months on the diet in all cases investigated. The long-term efficacy of the MAD in our patients is shown in Figure 3. The responder rate was 4/8 (50\%) after 3 months, 3/6 (50\%) after 6 months, 3/4 (75\%) after 12 months, and 2/4 (50\%) after 24 months on the diet. All of 4 patients who completed 24 months of the diet discontinued the diet afterwards. One patient (case2) of those who achieved seizure control at the 24 months has been seizure-free since then, although the other patient (case-3) suffered a relapse of seizures soon after the diet was stopped. There were no side effects on the laboratory examinations during the course of the diet except serum total-cholesterol which were slightly elevated (270$300 \mathrm{mg} / \mathrm{dl}$ ) in some patients. There were no patients who experienced weight loss or failure of appropriate weight gain during the diet. Severe side effects, which led to an interruption of the MAD occurred in 3 patients: generalized fatigue with severe acidosis ( $\mathrm{pH} 7.20$ on blood gas analysis) on the third day of the diet (case-2). Generalized fatigue with unknown cause within 1 week on the diet (case-9), and fainting with hypoglycemia due to refusal of food intake after one month on the diet (case-16). Although case- 9 and -16 gave up the restart of the MAD, case- 2 could resume the MAD ten days later. Our results suggest that even the MAD has potentially severe life-threatening side effects, especially in the early period from the start of the diet, although many authors have emphasized that the MAD can be safely introduced without hospital admission in contrast to the classical KD [1].

\section{The Low Glycemic Index Treatment (LGIT)}

\section{Dietary concept, composition and menu}

The glycemic index (GI), designed by Jenkins in 1981 [2], is an indicator of the degree of the increase of blood glucose levels by a specific food as compared to that of a standard food. Low GI foods have since been used for the treatment of diabetes mellitus and obesity.
In 2005, Pfeifer et al. from Massachusetts General Hospital first reported on an alternative ketogenic diet, 'LGIT' for the purpose of maintaining compliance to diet therapy [18]. This diet allows a more liberal daily intake of carbohydrates (40-60 g per day) than the other $\mathrm{KDs}$, on condition that the GI of carbohydrates is restricted to less than 50 relative to glucose. In the LGIT, a typical proportions of calories are as follows; $60-65 \%$ from fat, $20-30 \%$ from protein, and $10-15 \%$ from carbohydrates (Figure 1). Calories and fluids are not restricted. As a result, the diet is a more palatable and less rigid $\mathrm{KD}$ (ketogenic ratio is almost 1:1). The foods do not need to be weighed since the amount is based on portion sizes, which are based on diabetic exchanges.

The GI values of foods available in Western countries can be found in the literature [27]. In addition to this report, we used the list of the GI values of Japanese foods prescribed in our previous report [28]. Japanese ethnic foods such as $u$ don (flour noodle), soba (buckwheet noodle), and unpolished Japonica rice mixed with various supplements such as natto (fermented soybeans) and grated yam are all available in the LGIT.

Figure $2 c$ shows an example of a lunch menu for the LGIT used in our hospital, in which the total daily calorie intake is $1600 \mathrm{kcal}$. The carbohydrate used in the lunch was unpolished Japonica rice mixed with natto. The GI value of the unpolished rice is lower than that of polished rice because it has more fiber than its polished counterpart, which can delay the absorption of glucose from the intestine and decrease the speed at which blood glucose increases. Moreover, when mixed with natto, the rate of glucose absorption was reduced, and the GI value of unpolished rice decreases further to fewer than 50 relative to glucose. The content of carbohydrates, protein, and fat in Figure 1c was $22.2 \mathrm{~g}, 19.3 \mathrm{~g}$, and $39.5 \mathrm{~g}$ respectively, and the ketogenic ratio was $0.95: 1$, which is lower than that of the classical KD and the MAD.

\section{Evidence of efficacy based on previous literatures}

The LGIT was first reported to be effective for the treatment of medication-resistant epilepsy at Massachusetts General Hospital [18]. In their recent report, the responder rate was $42 \%, 50 \%, 54 \% 64 \%$, and $66 \%$ at $1,3,6,9$ and 12 months after treatment, respectively in intentto-treat analysis [29]. Coppola et al. from Italy showed that 8 of 15 
Citation: Kumada T, Miyajima T, Hiejima I, Nozaki F, Hayashi A, et al. (2013) Modified Atkins Diet and Low Glycemic Index Treatment for MedicationResistant Epilepsy: Current Trends in Ketogenic Diet. J Neurol Neurophysiol S2: 007 doi:10.4172/2155-9562.S2-007

patients (53\%) with the LGIT achieved $>50 \%$ seizure reduction in the first report published outside of Massachusetts General Hospital [30]. Recently, successful treatments with the LGIT for seizures associated with tuberous sclerosis complex [31], Angelman syndrome [32], and mitochondrial disorder [33] have also been reported. However, no controlled studies on the efficacy of the LGIT for the treatment of medication-resistant epilepsy are available. To qualify the evidence for the LGIT, randomized controlled studies are necessary.

\section{Our experience}

We first introduced the LGIT to case-13 in Table 1 because she had refused to comply with the MAD only 2 weeks after the diet therapy even though her seizures remitted with the MAD. She continued to tolerate the LGIT for over 1 year and achieved $>50 \%$ seizure reduction. Her detailed clinical course with this diet therapy will be published elsewhere [28]. We have tried the LGIT in only 3 patients and no reports other than ours are available in Japan so far. It seems, therefore, that the LGIT has not yet become a popular therapy for medicationresistant epilepsy in Japan. However, we assume that this diet will gain popularity in this country where the rice is a staple food; rice is acceptable with this diet therapy although only unpolished and a small amount of rice were permitted.

\section{Which diet should we use, the MAD or the LGIT?}

Several reports in the literature compared the efficacy of the classical KD and the MAD: Two articles reported a tendency for a higher responder rate with the classical $\mathrm{KD}$ than the MAD after 6 months (7/17 vs. $2 / 10, \mathrm{p}=0.41$ in Porta et al. [11], 30/50 vs. $13 / 33, \mathrm{p}=0.06$ in Miranda et al. [34]), although the differences were not significant. Kossoff et al. reported that patients with Doose syndrome achieved more seizure control by switching from the MAD to the classical KD [35]. In addition, Auvin insisted that the classical KD, not the MAD

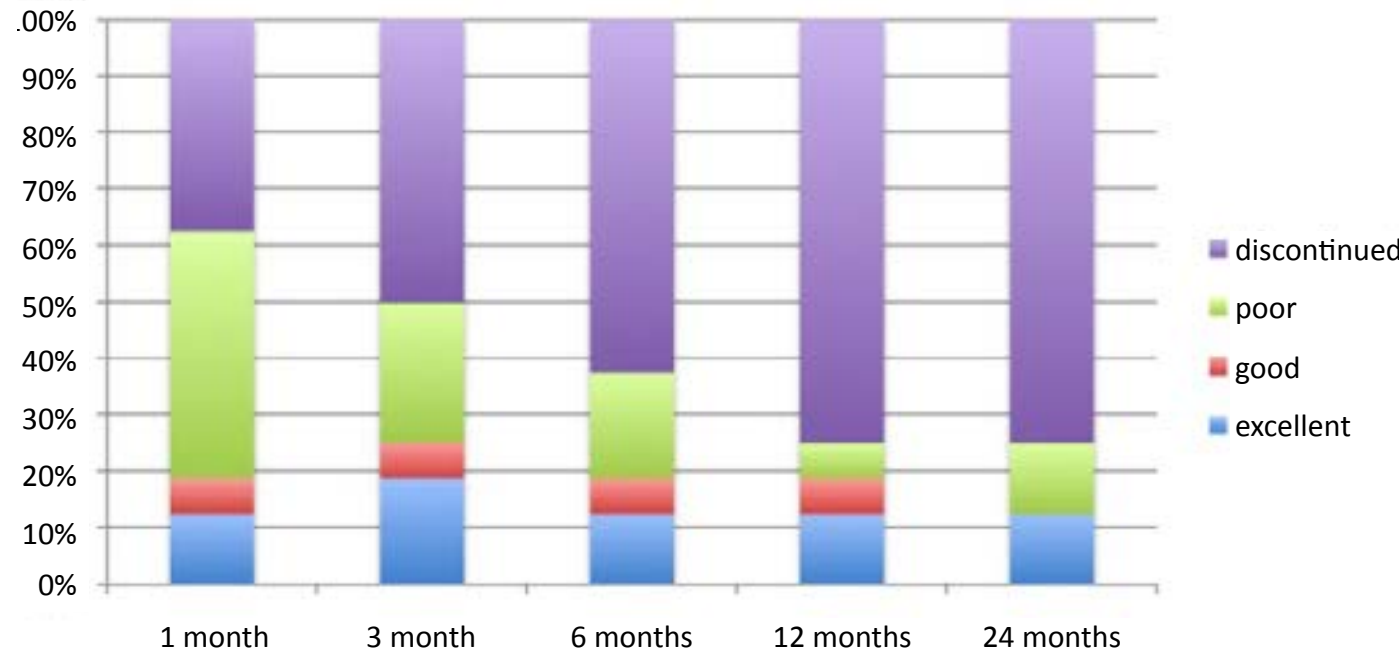

Figure 3: Percent of patients with excellent response (seizure-free), good response ( $>50 \%$ seizure reduction), poor response ( $<50 \%$ seizure reduction) on LGIT, and discontinued at each follow up interval.

\begin{tabular}{|c|c|c|c|c|c|c|c|c|}
\hline Case/sex & Age at the & Underlying & Epilepsy & Seizure & Seizure & Efficacy & Reasons of & beta-hydroxy- \\
\hline & start of MAD & disease & classification & phenotype & frequency & at 3 months & discontinuation & butyrate at 3 months \\
\hline 1/Male & $1 \mathrm{y} 6 \mathrm{~m}$ & leukodystrophy & SLRE & Complex Partial & Weekly & Discontinued & Reject By Patient & - \\
\hline 2/Female & $1 \mathrm{y} 6 \mathrm{~m}$ & Trisomy 21 & IS & Spasms & Daily & Excellent & & $4929 \mathrm{umol} / \mathrm{L}$ \\
\hline 3/Male & $1 \mathrm{y} 11 \mathrm{~m}$ & & IS & Spasms, Tonic & Daily & Excellent & & $3986 \mathrm{umol} / \mathrm{L}$ \\
\hline 4/Male & $2 y 2 m$ & & IS & Spasms, Tonic & Daily & Unchanged & & $5470 \mathrm{umol} / \mathrm{L}$ \\
\hline 5/Female & $3 y$ & & Doose & MAS, Myoclonic & Daily & Discontinued & Reject By Family & - \\
\hline 6/Female & $3 y$ & & IS & Spasms, Tonic & Daily & Unchanged & & not done \\
\hline 7/Female & $3 y$ & & IS & Spasms, Tonic & Daily & Unchanged & & not done \\
\hline 8/Male & $3 y$ & & IS & Spasms, Tonic & Daily & Unchanged & & 6174 umol/L \\
\hline 9/Female & $3 y$ & tuberous sclerosis & LGS & Tonic, Atypical Absence & Daily & Discontinued & Reject By Patient & - \\
\hline 10/Female & $5 y$ & band heterotopia & unclassified & NCSE, Gtcs, Drop & Daily & Excellent & & $2376 \mathrm{umol} / \mathrm{L}$ \\
\hline 11/Female & $5 y$ & & SLRE & NCSE, Tonic, Hypermotor & Daily & Good & & $2795 \mathrm{umol} / \mathrm{L}$ \\
\hline 12/Male & $7 y$ & & SLRE & Tonic & Weekly & Discontinued & Reject By Family & - \\
\hline 13/Female & $11 y$ & tuberous sclerosis & LGS & Tonic, Atypical Absence & Daily & Discontinued & Reject By Patient & - \\
\hline 14/Female & $17 y$ & tuberous sclerosis & LGS & Tonic, Atypical Absence & Daily & Discontinued & Reject By Family & - \\
\hline 15/Female & $29 y$ & $\begin{array}{l}15 q \text { inv dup } \\
\text { syndrome }\end{array}$ & SLRE & & Daily & Discontinued & Reject By Family & - \\
\hline 16/Female & $32 y$ & & SLRE & Complex Partial, Gtcs & Weekly & Discontinued & Reject By Patient & - \\
\hline
\end{tabular}

Table 1: Profiles of patients who started the MAD in our hospital and the short-term efficacy and tolerability after 3 months on the diet Abbreviations: SLRE, symptomatic localization-related epilepsy; IS, infantile spasms; LGS, Lennox-Gastaut syndrome; Doose, Doose syndrome; NCSE, nonconvulsive status epilepticus; GTCs, generalized tonic clonic seizure; excellent, seizure-free; good, $>50 \%$ seizure reduction; unchanged; $<50 \%$ seizure reduction. 


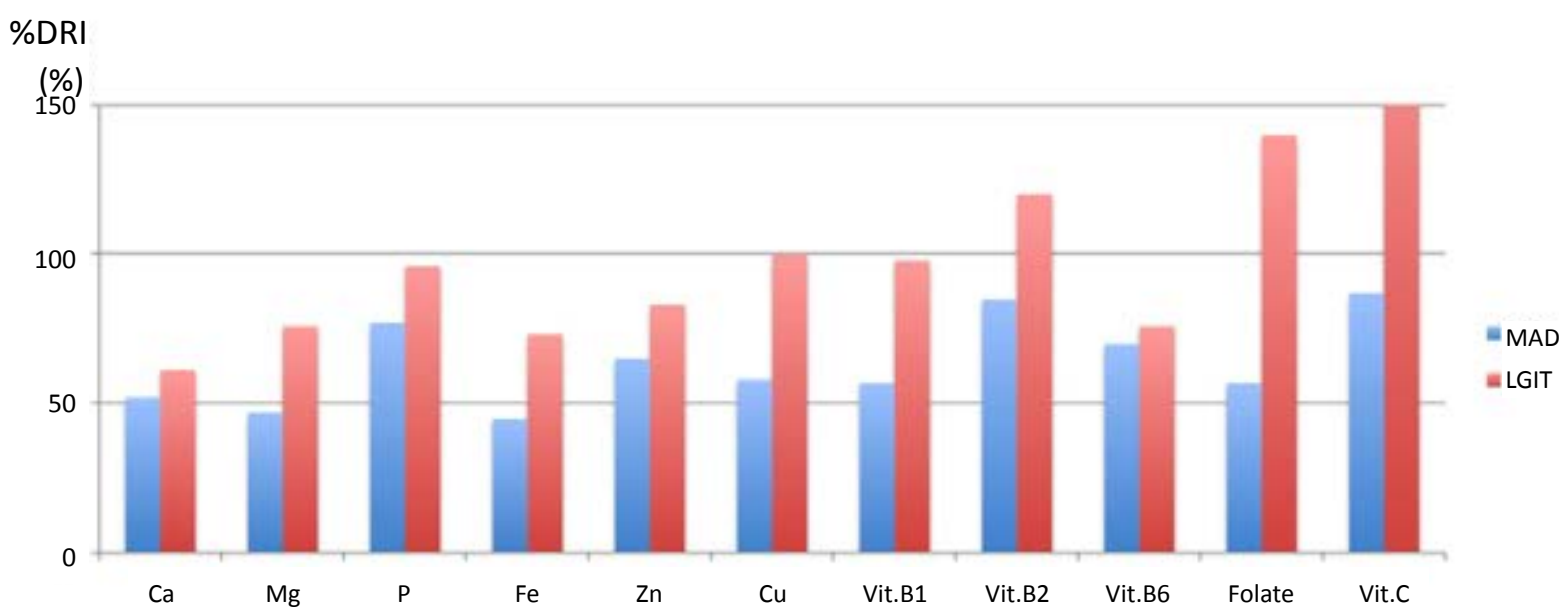

Figure 4: \%DRI of daily micronutrient distribution based on the mean amount in each of the diets (the MAD and the LGIT) prepared for case-13 at our hospital. \%DRI was calculated as the daily amount of each micronutrient (the mean value for 2 weeks) divided by the recommended dietary allowance provided by 'Dietary reference intakes for Japanese, 2010'. Based on this, insufficiency in intake of a specific micronutrient resulted in a \%DRI $<100 \%$. Blue rod: the MAD, Red rod: the LGIT

should be tried in the epileptic emergency such as recent onset of infantile spasms and refractory status epilepticus because the former can resolve the seizures faster [20].

Although increasing number of evidences are becoming available that indicates an appropriate choice between the classical KD and the MAD as mentioned above, we still do not have clear answers to govern our choice between the MAD or the LGIT. Our experience with case13 in Table 1 who tolerated the LGIT better than the MAD suggested that the LGIT should be considered for patients with medicationresistant epilepsy who cannot tolerate more restrictive diet therapies. On the other hand, there has not been any comparative data from the dietetic perspective between the MAD and the LGIT. Figure 4 shows the micronutrient distribution in the MAD and the LGIT given to case-13 at our hospital. The \% dietary reference intakes (\%DRI) of all of the minerals and soluble vitamins listed were higher in the LGIT than in the MAD. Furthermore, among all of the subjects, the \%DRI of some soluble vitamins (B2, C, and folate) was $>100 \%$ in the LGIT, making supplementation unnecessary. Therefore, we believe that the LGIT would be superior to the MAD based on its tolerability and dietetic balance. However, we do not have data from a controlled trial or retrospective analysis, comparing the efficacy of the MAD and the LGIT.

\section{Conclusion}

Currently, there is a large volume of evidence, including one controlled trial, on the efficacy of the MAD for seizure control, and the diet was established as an important treatment for medicationresistant epilepsy. In contrast, there is less evidence for use of the LGIT without any controlled trials. This therapy is less popular than the MAD, especially in Japan. Further comparative data on the efficacy, tolerability and dietetic balance of both the MAD and the LGIT are needed in order to select the best type of diet therapy for patients with medication-resistant epilepsy.

\section{Acknowledgements}

We gratefully thank Miyuki Takada, the dietitian of our hospital, for designing the MAD and LGIT menus. The authors report no conflicts of interest.

\section{References}

1. Kossoff EH, Dorward JL (2008) The modified Atkins diet. Epilepsia 49 Suppl 8: $37-41$.
2. Pfeifer HH, Lyczkowski DA, Thiele EA (2008) Low glycemic index treatment: implementation and new insights into efficacy. Epilepsia 49 Suppl 8: 42-45.

3. Wheless JW (2004) Epilepsy and the ketogenic diet. Humana Press, New Jersey.

4. Freeman JM, Kossoff EH, Hartman AL (2007) The ketogenic diet: one decade later. Pediatrics 119: 535-543.

5. Kossoff EH, Zupec-Kania BA, Amark PE, Ballaban-Gil KR, Christina Bergqvist $A G$, et al. (2009) Optimal clinical management of children receiving the ketogenic diet: recommendations of the International Ketogenic Diet Study Group. Epilepsia 50: 304-317.

6. Kossoff EH, Krauss GL, McGrogan JR, Freeman JM (2003) Efficacy of the Atkins diet as therapy for intractable epilepsy. Neurology 61: 1789-1791.

7. Kossoff EH, McGrogan JR, Bluml RM, Pillas DJ, Rubenstein JE, et al. (2006) A modified Atkins diet is effective for the treatment of intractable pediatric epilepsy. Epilepsia 47: 421-424.

8. Kossoff EH, Rowley H, Sinha SR, Vining EP (2008) A prospective study of the modified Atkins diet for intractable epilepsy in adults. Epilepsia 49: 316-319.

9. Weber S, Mølgaard C, Karentaudorf, Uldall P (2009) Modified Atkins diet to children and adolescents with medical intractable epilepsy. Seizure 18: 237 240 .

10. Miranda MJ, Mortensen M, Povlsen JH, Nielsen H, Beniczky S (2011) Danish study of a modified Atkins diet for medically intractable epilepsy in children: can we achieve the same results as with the classical ketogenic diet? Seizure 20: 151-155.

11. Porta N, Vallée L, Boutry E, Fontaine M, Dessein AF, et al. (2009) Comparison of seizure reduction and serum fatty acid levels after receiving the ketogenic and modified Atkins diet. Seizure 18: 359-364.

12. Carrette E, Vonck K, de Herdt V, Dewaele I, Raedt R, et al. (2008) A pilot tria with modified Atkins' diet in adult patients with refractory epilepsy. Clin Neuro Neurosurg 110: 797-803

13. Kang HC, Lee HS, You SJ, Kang du C, Ko TS, et al. (2007) Use of a modified Atkins diet in intractable childhood epilepsy. Epilepsia 48: 182-186.

14. Kim YM, Vaidya VV, Khusainov T, Kim HD, Kim SH, et al. (2012) Various indications for a modified Atkins diet in intractable childhood epilepsy. Brain Dev 34: 570-575.

15. Sharma S, Sankhyan N, Gulati S, Agarwala A (2012) Use of the modified Atkins diet in infantile spasms refractory to first-line treatment. Seizure 21: 45-48.

16. Tonekaboni $\mathrm{SH}$, Mostaghimi $\mathrm{P}$, Mirmiran $\mathrm{P}$, Abbaskhanian $\mathrm{A}$, Abdollah Gorj $F$, et al. (2010) Efficacy of the Atkins diet as therapy for intractable epilepsy in children. Arch Iran Med 13: 492-497.

17. Kumada T, Miyajima T, Oda N, Shimomura H, Saito K, et al. (2012) Efficacy 
Citation: Kumada T, Miyajima T, Hiejima I, Nozaki F, Hayashi A, et al. (2013) Modified Atkins Diet and Low Glycemic Index Treatment for MedicationResistant Epilepsy: Current Trends in Ketogenic Diet. J Neurol Neurophysiol S2: 007 doi:10.4172/2155-9562.S2-007

and tolerability of modified Atkins diet in Japanese children with medicationresistant epilepsy. Brain Dev 34: 32-38.

18. Pfeifer $\mathrm{HH}$, Thiele EA (2005) Low-glycemic-index treatment: a liberalized ketogenic diet for treatment of intractable epilepsy. Neurology 65: 1810-1812.

19. Payne NE, Cross JH, Sander JW, Sisodiya SM (2011) The ketogenic and related diets in adolescents and adults--a review. Epilepsia 52: 1941-1948.

20. Auvin S (2012) Should we routinely use modified Atkins diet instead of regular ketogenic diet to treat children with epilepsy? Seizure 21: 237-240.

21. Chen W, Kossoff EH (2012) Long-term follow-up of children treated with the modified Atkins diet. J Child Neurol 27: 754-758.

22. Sharma S, Sankhyan N, Gulati S, Agarwala A (2013) Use of the modified Atkins diet for treatment of refractory childhood epilepsy: a randomized controlled trial. Epilepsia 54: 481-486.

23. Groomes LB, Pyzik PL, Turner Z, Dorward JL, Goode VH, et al. (2011) Do patients with absence epilepsy respond to ketogenic diets? J Child Neurol 26: 160-165.

24. Kossoff EH, Henry BJ, Cervenka MC (2013) Efficacy of dietary therapy for juvenile myoclonic epilepsy. Epilepsy Behav 26: 162-164.

25. Kossoff EH, Borsage JL, Comi AM (2010) A pilot study of the modified Atkins diet for Sturge-Weber syndrome. Epilepsy Res 92: 240-243.

26. Kumada T, Miyajima T, Kimura N, Saito K, Shimomura H, et al. (2010) Modified Atkins diet for the treatment of nonconvulsive status epilepticus in children. $J$ Child Neurol 25: 485-489.
27. Foster-Powell K, Holt SH, Brand-Miller JC (2002) International table of glycemic index and glycemic load values: 2002. Am J Clin Nutr 76: 5-56.

28. Kumada T, Hiejima I, Nozaki F, Hayashi A, Fujii T (2013) Glycemic index treatment using Japanese foods in a girl with Lennox-Gastaut syndrome. Pediatr Neurol 48: 390-392.

29. Muzykewicz DA, Lyczkowski DA, Memon N, Conant KD, Pfeifer $\mathrm{HH}$, et al. (2009) Efficacy, safety, and tolerability of the low glycemic index treatment in pediatric epilepsy. Epilepsia 50: 1118-1126.

30. Coppola G, D'Aniello A, Messana T, Di Pasquale F, della Corte R, et al. (2011) Low glycemic index diet in children and young adults with refractory epilepsy: first Italian experience. Seizure 20: 526-528

31. Larson AM, Pfeifer HH, Thiele EA (2012) Low glycemic index treatment for epilepsy in tuberous sclerosis complex. Epilepsy Res 99: 180-182.

32. Thibert RL, Pfeifer HH, Larson AM, Raby AR, Reynolds AA, et al. (2012) Low glycemic index treatment for seizures in Angelman syndrome. Epilepsia 53: 1498-1502.

33. Martikainen MH, Päivärinta $M$, Jääskeläinen S, Majamaa K (2012) Successful treatment of POLG-related mitochondrial epilepsy with antiepileptic drugs and low glycaemic index diet. Epileptic Disord 14: 438-441.

34. Miranda MJ, Turner Z, Magrath G (2012) Alternative diets to the classical ketogenic diet--can we be more liberal? Epilepsy Res 100: 278-285.

35. Kossoff EH, Bosarge JL, Miranda MJ, Wiemer-Kruel A, Kang HC, et al. (2010) Will seizure control improve by switching from the modified Atkins diet to the traditional ketogenic diet? Epilepsia 51: 2496-2499.
This article was originally published in a special issue, Epilepsy: Current Trends handled by Editor(s). Dr. Espinosa PS, Centro Internacional en Neurociencias, USA 